\title{
Identification of 11 novel and common single nucleotide polymorphisms in the interleukin-7 receptor- $\alpha$ gene and their associations with multiple sclerosis
}

\author{
Suzy M Teutsch ${ }^{* 1,2}$, David R Booth ${ }^{1,2}$, Bruce H Bennetts ${ }^{3}$, Robert NS Heard ${ }^{1,2}$ and \\ Graeme J Stewart ${ }^{1,2}$
}

\footnotetext{
${ }^{1}$ Institute for Immunology and Allergy Research (Westmead Millennium Institute), Westmead Hospital, Westmead, NSW 2145, Australia; ${ }^{2}$ The University of Sydney, NSW 2006, Australia; ${ }^{3}$ Department of Molecular Genetics, The Children's Hospital at Westmead, Westmead, NSW 2145, Australia
}

We have investigated the interleukin-7 receptor (IL-7R) $\alpha$-chain gene as a positional and functional candidate gene for susceptibility to multiple sclerosis (MS), in view of its chromosomal location on 5p14-p12, a region that has shown suggestive linkage in MS genome screens, and its role in T- and B-cell proliferation and reactivity. Amplification and DNA sequencing of the IL-7R $\alpha$ gene in pooled and individual samples identified 13 single nucleotide polymorphisms (SNPs), 11 of which are novel, including three in the promoter region, three in exons encoding amino-acid changes (ACC(Thr)66ATC(Ile),

ATC(Ile)244ACC(Thr), ATC(Ile)336GTC(Val)), four in introns and one in the $3^{\prime}$ untranslated region. Four IL-7R haplotypes were identified for nine SNPs, showing linkage disequilibrium across the gene, and allowing haplotype frequency determination from just three of the nine SNPs. Genotyping of the -504 polymorphism in $101 \mathrm{MS}$ and 90 controls showed a suggestive $(P=0.1)$ association of the T allele with MS; however, this was not supported by transmission disequilibrium testing in $186 \mathrm{MS}$ trio families $(P=0.8)$. There were trends towards an increase of the $G T G+$ haplotype (odds ratio $=1.45$ ), and underrepresentation of the TTA+ haplotype $(O R=0.65)$ in DRB1*1501-positive MS cases, suggesting that larger sample sizes and comparison in more defined MS patient groups may support an association with the IL-7R gene. These polymorphisms would also be useful for studying genetic associations with other immunologic diseases.

European Journal of Human Genetics (2003) 11, 509-515. doi:10.1038/sj.ejhg.5200994

Keywords: interleukin-7 receptor $\alpha$; single nucleotide polymorphism; haplotype; multiple sclerosis

\section{Introduction}

Multiple sclerosis (MS) is the most common chronic (inflammatory) disease of the central nervous system

\footnotetext{
*Correspondence: SM Teutsch, Institute for Immunology and Allergy Research, Level 2, ICPMR, Westmead Hospital, Westmead NSW 2145, Australia. Tel: +61 29845 6791; Fax: +61 29891 3889;

E-mail: suzyt@westgate.wh.usyd.edu.au

Received 23 July 2002; revised 17 February 2003; accepted 20 February 2003
}

affecting young adults. Although the pathogenesis has yet to be elucidated, there is increasing evidence that MS is the result of an interaction of genetic and environmental factors. ${ }^{1}$ While MS genome screens have not identified a single MS susceptibility locus, multiple chromosomal regions showing suggestive linkage have been observed, ${ }^{2-7}$ indicating that MS susceptibility may be determined by the interaction of multiple genes exerting modest effects. ${ }^{8}$

The chromosome $6 \mathrm{p} 21$ region, harbouring genes of the major histocompatibility complex, was identified across all 
studies as a major region of susceptibility, and several other chromosomal regions were identified as warranting further investigation, notably including 5p14-p12. Positive linkage with this region was detected in a Canadian MS genome screen, ${ }^{4}$ and in a Scandinavian ${ }^{9}$ study of chromosome $5 p$ markers. This region has also been found to be syntenic with experimental autoimmune encephalomyelitis (EAE) susceptibility loci, ${ }^{10}$ supported by positive linkage with these chromosomal regions in Finnish MS families. ${ }^{11}$

Attempts to identify candidate genes for MS susceptibility within the chromosome 5p14-p12 region have already begun, with one report finding no significant associations with the complement factors C6 and C7. ${ }^{12}$ Another gene encoded in this region is the interleukin-7 receptor (IL-7R) $\alpha$-chain (CD127) gene. IL-7R is the receptor for IL-7, an important T-cell growth factor in T- and B-cell expansion. ${ }^{13,14}$ It consists of the $\alpha$-chain, in combination with the common interleukin-2 receptor (IL-2R) $\gamma$-chain. ${ }^{15}$ Differential splicing of mRNA transcripts ${ }^{13}$ has identified membrane-bound and soluble forms of the protein.

Both IL-7 and its receptor have been implicated in MS pathogenesis. IL-7 has been shown to increase T-cell proliferation $^{16}$ and reactivity ${ }^{17}$ to myelin antigens in MS and in EAE. ${ }^{18}$ In the latter study, IL-7 expanded T cells were also found to cause more severe EAE when adoptively transferred to naïve recipient mice. More recently, a microarray study showed IL-7R cDNA expression to be significantly increased in PBMCs from MS patients with relapsing-remitting disease, compared with normal controls. $^{19}$

The chromosomal location of the IL-7R gene, together with the potential functional role of the IL-7R molecule in MS pathogenesis, provide a strong case for this gene to be investigated as a positional candidate gene for MS susceptibility. It has recently been proposed that examining the haplotypes between single nucleotide polymorphisms (SNPs) may be a more effective way of mapping disease susceptibility genes than examining associations with individual SNPs alone. ${ }^{20,21}$ In this study, the IL-7R $\alpha$-chain gene was screened for SNPs in order to construct haplotypes, which could be used to examine associations with MS susceptibility in Australian patients.

\section{Patients and methods}

\section{MS patients and control subjects}

The patient groups consisted of unrelated MS cases, MS trio families (patient and both biological parents) and probands from MS multiplex families. Only patients with clinically definite or laboratory-supported definite MS, according to the diagnostic criteria of Poser et $a l^{22}$ were studied. Ethnically matched unrelated control subjects were randomly selected from hospital staff and unrelated partners of MS subjects. All subjects were Caucasian, with $90 \%$ of Northern European origin.

\section{Construction of DNA pools}

DNA samples from 217 DR2-positive MS patients, 155 DR2negative MS patients, 169 probands from MS multiplex families and from 187 unrelated controls were pooled separately using a protocol based on previously described methods. ${ }^{23}$ DNA samples were mixed with PicoGreen reagent (Molecular Probes, Eugene, OR, USA) in 96-well Titertek black plates (ICN, Aurora, OH, USA) and fluorescence was measured using the FluoroCount fluorescence microplate reader (Packard BioScience, Meriden, CT, USA). Concentrations were determined by construction of a standard curve. Samples were diluted to $100 \mathrm{ng} / \mu \mathrm{l}$ and measured at least twice, before diluting to a final concentration of $50 \mathrm{ng} / \mu \mathrm{l}$. A volume of $100 \mu \mathrm{l}(5 \mu \mathrm{g})$ of each sample was pooled and vortexed in a $50 \mathrm{ml}$ tube. Pools were stored at $-20^{\circ} \mathrm{C}$.

\section{PCR amplification}

PCR primers were designed using the GenBank accession numbers for IL-7R genomic DNA sequences described in Puel et al. ${ }^{24}$ A fragment of the IL-7R $\alpha$ gene promoter region (nucleotides -280 to -1348 ) was amplified in genomic DNA samples from 17 MS individuals using the following PCR primer sequences: IL-7R1 5'-ATACCTAGGCACTAATT TAG and IL-7R2 3'-CCCACACTTACTGTGCTTACATCAGAG. The following sets of PCR primers were used to amplify the rest of the IL7R gene in pooled and individual DNA samples: promoter region (nucleotides -280 to 0 ), p-F: 5'-TAGCCTCTAGCCTAAGATAGCTT and p-R: $3^{\prime}$ TTTCTCCAGAAACGACTTGAA GT; exon 1, p-F and X1R2: 3'-TAACCTGAAACCATGCTACAGAT; exon 2, X2F: 5'-ATGTCTGCCACAGAGTCTGCT and X2R: 3'-GCCTTT GGGAATTCCTTAGATG; exon 3, X3F: 5'-CCTACCTGAAT CAAGACATACC and X3R: 3'-TGCACAAA CACTATCTTT CACAC; exon 4, X4F: 5'-TATTTCCTTGGCTGCCCTTTAG and X4R: $3^{\prime}$-GAAATGCACTACTAGGCCAACA; exon 5, X5F: 5'-CGAGACAACTTC AGAGAATGCT and X5R: 3'-CAATCTTCСТCTCACTTGCTCC; exon 6, X6F: 5'CTTCAAGTGGCAGATGCTCTG and X6R: 3'-CCCACACAA TCACCCTCTTTAT; exon 7, X7F: 5'-TTGATATCTGTGGT CTCTGGTC and X7R: 3'-TACTG CAGCTAGGGTCTTAGG; exon 8, X8F: 5'-TCTGAGAAGCAGAGGCTTGGA and X8R: 3'-GGGAGACTAGGAACTCTAGAC. Standard PCR conditions were used.

\section{DNA sequencing}

PCR products were purified by using the Qiaquick PCR product purification kit (Qiagen GmbH, Hilden, Germany) or by polyethylene glycol precipitation (nucleotides -280 to -1348 of the promoter). DNA sequencing was performed using the ABI Prism Big Dye Terminator version 3.0 or version 1.0 (nucleotides -280 to -1348 of the promoter) sequencing kit (Applied Biosystems, Foster City, CA, USA) and an ABI 3100 machine. PCR products were sequenced in one direction using the following internal primers (100 ng 
used per reaction): promoter, P-I: 5'-GCCTAAGATAGCTT GAGCCT; exon 1, P-I; exon 2, X2I: 5'GCCACAGAGTC TGCTATTTTATT; exon 3 (primer in reverse direction), X3I: 3'-CC TGGGTTTGAAGATCCTCAG; exon 4 (primer in reverse direction), X4I: 3'-GCCAACAAAATAGAAAACATTC; exon 5, X5I: 5'-TGCTTATGGGACTAAAGG AATTC; exon 6, X6I: 5'-CCTGGTCACCCAAGTCAATG; exon 7, X7I: 5'TCCAACCCCTCCTTGAATTG; exon 8, X8I: 5'-AGAGG CTTGGAGGGGATGTG. PCR products were also sequenced in the alternate direction using the appropriate PCR primer.

\section{PCR-RFLP genotyping of IL-7R promoter region and} exon 8 codon 336 SNPs

PCR products were digested with PstI to detect the -504 (T/ C) promoter polymorphism, with $H p h$ I to detect the -1085 $(\mathrm{G} / \mathrm{T})$ and -449 (A/G) promoter polymorphisms, and with Tsp45I to detect the exon 8 codon 336 (A/G) polymorphism, according to the manufacturer's directions (NEB, Beverly, MA, USA).

\section{Genotyping of IL-7R promoter region SNPs by SNaPshot}

Genotyping of additional MS cases and controls was performed by initial PCR amplification with the IL-7R1 primer and a modified IL-7R2 primer (3'-CCCACACT TACTGTGCTTACATC). Three extension primers were designed for promoter SNPs: IL-7R-1085: 5'- TTTGTAG ATGATACACAAATGGGT, IL-7R-504: 5' - TTTTTTTTTGG CATAGTG GCATTTGCCTG, IL-7R-449: 5'- TGCTTGAA
CCTGGGAGGTG. SNaPshot reactions were analysed as described by the manufacturer (ABI PRISM SNaPshot Multiplex Kit, Applied Biosystems, Foster City, CA, USA).

\section{Statistical analysis}

Associations between IL-7R promoter alleles and MS were examined in case/controls using $\chi^{2}$ analysis, and in MS trio families using transmission disequilibrium test (TDT) analysis. $^{25}$ The SPSS statistical package (SPSS Inc., Chicago, IL, USA) was used to determine $P$-values.

\section{Results}

Identification of SNPs in the IL-7R $\alpha$ gene

DNA sequencing of the IL-7R $\alpha$ gene promoter and exons 1-8 in pooled and individual samples identified 13 SNPs: three in the promoter region, five in exons, four in introns and one in the $3^{\prime}$ untranslated region (Figure. 1). All SNPs were confirmed by forward and reverse sequencing in heterozygotes. The codon ATC(Ile)118 $\rightarrow$ GTC(Val) and CAC(His) $145 \rightarrow$ CAT(His) SNPs in exon 4 were identical to those recorded in the dbSNP database (http:// www.ncbi.nlm.nih.gov/SNP/); however, the reported exon 8 polymorphisms in codons 339 and 394 were not detected. The remaining 11 SNPs were novel, with the exon 2 ACC(Thr) $66 \rightarrow$ ATC(Ile), and exon 6 ATC(Ile) $244 \rightarrow$ ACC(Thr) polymorphisms producing nonconservative amino-acid changes, and the exon 8 ATC(Ile)336 $\rightarrow$

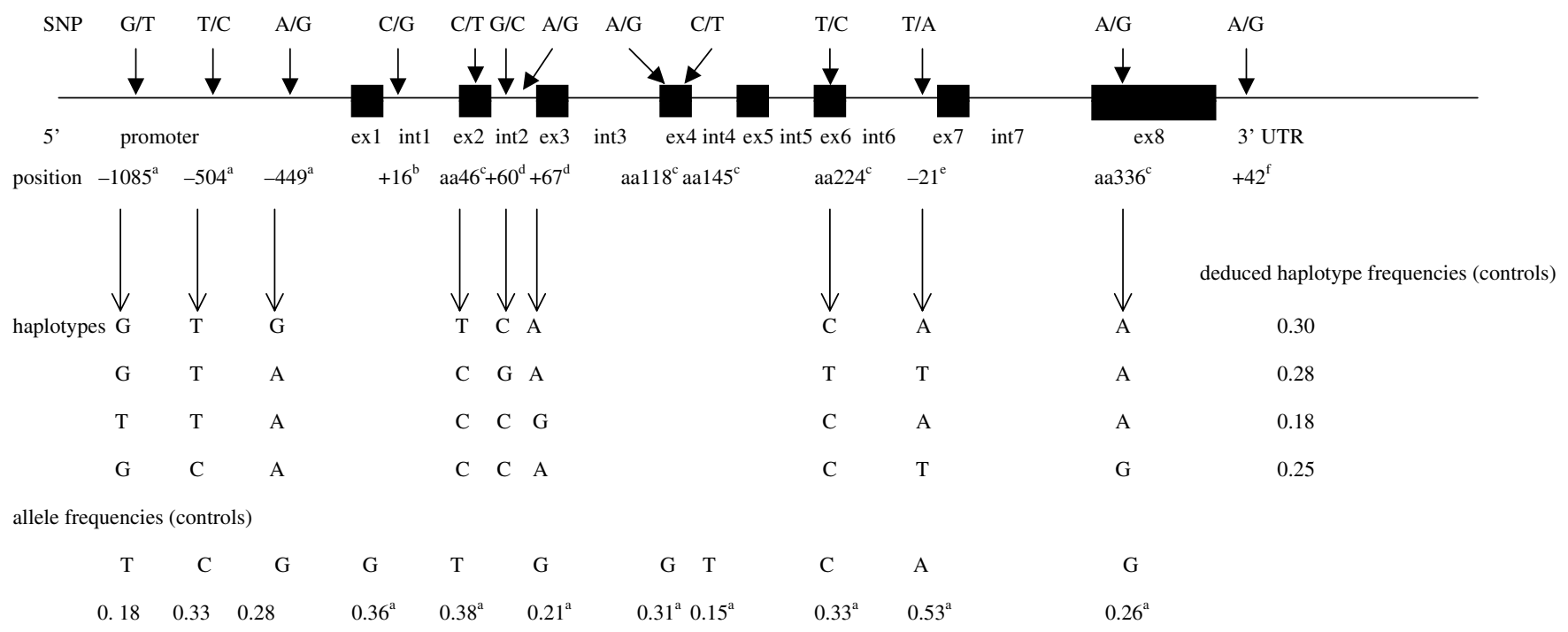

a estimate determined from pooled DNA sequencing

Figure 1 Schematic diagram of IL-7R genomic sequence showing SNPs identified by pooled and individual DNA sequencing, and four identified haplotypes. a Minus the number of nucleotides from the start of exon 1 (GenBank accession number AF043123). ${ }^{b}$ Plus the number of nucleotides from the end of exon 1 (GenBank accession number AF043123). ${ }^{C}$ Numbering is from first amino acid residue in mature peptide. ${ }^{d}$ Plus the number of nucleotides from the end of exon 2 (GenBank accession number AF043124). ${ }^{e}$ Minus the number of nucleotides from the start of exon 7 (GenBank accession number AF043129). ${ }^{f}$ Plus the number of nucleotides from the end of exon 8 (GenBank accession number AF043129). 
GTC(Val) polymorphism producing a conservative aminoacid change.

Examination of the promoter region SNPs for potential transcription factor binding sites using the TRANSFAC program, ${ }^{26}$ showed a binding site for nuclear factor 1 to sequences containing the rarer $-1085 \mathrm{~T}$ allele, but not the $\mathrm{G}$ allele, and a site for nuclear factor for activated $\mathrm{T}$ cells and POU-factor Tst-1/Oct- 6 to sequences containing the rarer $-449 \mathrm{G}$ allele, but not the A allele. To examine whether the intron 6 and exon 6 SNPs lead to altered mRNA splicing of exon 6, which is excised to produce the soluble form of IL-7R, sequences were analysed using the Berkley Drosophila Genome Project Splice Site Prediction by Neural Network (http://www.fruitfly.org). However, no differences in the predicted splicing patterns were found for either of the polymorphisms.

\section{Genotyping of IL-7R promoter region SNPs and association testing with MS}

IL-7R genotyping of individual DNA samples was carried out using PCR-RFLP, SNaPshot and DNA sequencing methods. Table 1 shows the number of individuals in each dataset who were genotyped using these methods. PCRRFLP genotyping of the three promoter region polymorphisms in controls gave allele frequencies of 0.18 for the $-1085 \mathrm{~T}$ allele, 0.33 for the $-504 \mathrm{C}$ allele and 0.28 for the $-449 \mathrm{G}$ allele. Preliminary PstI genotyping of the -504 polymorphism (Table 2) showed a trend towards a significant difference in the allele and genotype frequencies in a group of unrelated MS cases and controls. In order to test this association further, 186 MS trio families were genotyped and analysed using the TDT, with no distortion observed in the transmission of either $\mathrm{C}$ or T alleles (79 vs 76) in 124 informative families (TDT statistic $=0.06$, $P=0.81)$.

\section{Determination of IL7R haplotypes and association testing with MS}

In all, 40 MS and control individuals, who were homozygous for the three promoter region SNPs by PCR-RFLP genotyping, were further genotyped for the exon 2 and intron 2 SNPs by sequencing, and for the exon 8 codon 336 (A/G) SNP by PCR-RFLP. All individuals homozygous for the promoter polymorphisms were homozygous at the

Table 1 Numbers of MS patients and controls IL-7R genotyped using PCR-RFLP, SNaPshot and DNA sequencing methods

\begin{tabular}{|c|c|c|c|c|c|c|c|c|}
\hline \multicolumn{4}{|l|}{ Test of analysis } & \multicolumn{3}{|c|}{$\begin{array}{l}\text { Number of individuals } \\
\text { in dataset }\end{array}$} & \multicolumn{2}{|c|}{$\begin{array}{l}\text { Genotyping } \\
\text { method }\end{array}$} \\
\hline \multirow{2}{*}{\multicolumn{4}{|c|}{$\begin{array}{l}\text { Case/control study of }-504 \\
\text { SNP associations }\end{array}$}} & \multicolumn{3}{|c|}{101 MS cases } & \multicolumn{2}{|c|}{ PCR-RFLP } \\
\hline & & & & \multicolumn{3}{|c|}{90 controls } & \multirow{2}{*}{\multicolumn{2}{|c|}{ PCR-RFLP }} \\
\hline \multicolumn{4}{|c|}{$\begin{array}{l}\text { Transmission disequilibrium testing } \\
\text { of }-504 \text { SNP associations }\end{array}$} & \multicolumn{3}{|c|}{$186 \mathrm{MS}$ trio probands } & & \\
\hline \multicolumn{9}{|c|}{$\begin{array}{l}\text { Determination of IL-7R haplotypes } \\
\text { in homozygous individuals: }\end{array}$} \\
\hline $\begin{array}{l}\text { Promoter SNPs } \\
\text { Exon } 2 \text { and intron } 2 \text { SNPs } \\
\text { Exon } 8 \text { codon } 336 \text { SNP }\end{array}$ & & & & \multicolumn{3}{|c|}{$\begin{array}{l}24 \mathrm{MS} \text { and } 16 \text { controls } \\
24 \mathrm{MS} \text { and } 16 \text { controls } \\
29 \mathrm{MS} \text { and } 18 \text { controls }\end{array}$} & \multicolumn{2}{|c|}{$\begin{array}{l}\text { PCR-RFLP } \\
\text { DNA sequencing } \\
\text { PCR-RFLP }\end{array}$} \\
\hline \multicolumn{4}{|c|}{$\begin{array}{l}\text { Case/control study of IL7-R haplotype } \\
\text { associations }\end{array}$} & \multicolumn{3}{|l|}{$176 \mathrm{MS}$} & \multicolumn{2}{|c|}{$\begin{array}{l}\text { PCR-RFLP } \\
\text { (95 MS, } 67 \text { controls) } \\
\text { SNaPshot } \\
\text { (81 MS, } 109 \text { controls) }\end{array}$} \\
\hline & & Genotype & Frequ & ncy & Allele & Frequency & Odds ratio & \\
\hline & $n$ & $T / T$ & $T / C$ & $\mathrm{C} / \mathrm{C}$ & $T$ & $C$ & $T+$ phenotype & $95 \% \mathrm{Cl}$ \\
\hline MS & 101 & $57(56 \%)$ & $37(37 \%)$ & $7(7 \%)$ & $151(0.7475)$ & $51(0.2525)$ & 1.310 & $0.409-4.229$ \\
\hline Controls & 90 & $\begin{array}{l}39(43 \%) \\
p=0.20\end{array}$ & $43(48 \%)$ & $8(9 \%)$ & $\begin{array}{l}121(0.6722), \\
p=0.11\end{array}$ & $59(0.3278)$ & & \\
\hline DRB1*1501+ MS vs controls & $57^{a}$ & $\begin{array}{l}31(54 \%) \\
p=0.27\end{array}$ & $24(42 \%)$ & $2(4 \%)$ & $\begin{array}{l}86(0.7544) \\
p=0.13\end{array}$ & $28(0.2456)$ & 2.683 & 0.499-19.061 \\
\hline DRB1*1501 - MS vs controls & $42^{a}$ & $\begin{array}{l}25(60 \%) \\
p=0.11\end{array}$ & $12(29 \%)$ & $5(12 \%)$ & $\begin{array}{l}62(0.7381) \\
p=0.28\end{array}$ & $22(0.2619)$ & 0.722 & $0.196-2.752$ \\
\hline
\end{tabular}

${ }^{\mathrm{a}} 99 \mathrm{MS}$ patients were stratified for the DRB1*1501 allele. 
Table 3 Comparison of IL-7R promoter region haplotype frequencies in MS patients and control individuals, and in HLADRB1*1501-positive and -negative individuals

\begin{tabular}{|c|c|c|c|c|c|c|c|}
\hline \multirow[b]{2}{*}{-1085} & \multicolumn{6}{|c|}{ Haplotype } & \multirow[b]{2}{*}{$95 \% \mathrm{Cl}$} \\
\hline & -504 & -449 & All MS $(2 N=352)$ & All controls $(2 \mathrm{~N}=352)$ & $P$ & Odds ratio & \\
\hline G & $\mathrm{T}$ & G & $117(33 \%)$ & $111(32 \%)$ & 0.69 & 1.081 & $0.778-1.501$ \\
\hline G & $\mathrm{T}$ & $A$ & $88(25 \%)$ & $92(26 \%)$ & 0.80 & 0.942 & $0.662-1.341$ \\
\hline $\mathrm{T}$ & $\mathrm{T}$ & A & $50(14 \%)$ & $55(16 \%)$ & 0.67 & 0.894 & $0.578-1.382$ \\
\hline \multirow[t]{2}{*}{ G } & $\mathrm{C}$ & A & $97(28 \%)$ & $94(27 \%)$ & 0.87 & 1.044 & $0.739-1.476$ \\
\hline & & & $\begin{array}{c}1501+M S^{a} \\
(2 N=144)\end{array}$ & $\begin{array}{c}1501+\text { Controls }^{a} \\
(2 N=100)\end{array}$ & $P$ & Odds ratio & $95 \% \mathrm{Cl}$ \\
\hline G & $\mathrm{T}$ & G & $60(42 \%)$ & $33(33 \%)$ & 0.22 & 1.450 & $0.823-2.560$ \\
\hline G & $\mathrm{T}$ & $A$ & $32(22 \%)$ & $22(22 \%)$ & 1.00 & 1.013 & $0.525-1.961$ \\
\hline $\mathrm{T}$ & $\mathrm{T}$ & A & 19 (13\%) & 19 (19\%) & 0.29 & 0.648 & $0.306-1.371$ \\
\hline \multirow[t]{2}{*}{ G } & $\mathrm{C}$ & A & $33(23 \%)$ & $26(26 \%)$ & 0.69 & 0.846 & $0.449-1.596$ \\
\hline & & & $\begin{array}{l}1501-M S \\
(2 N=116)\end{array}$ & $\begin{array}{c}1501-\text { Controls } \\
(2 \mathrm{~N}=210)\end{array}$ & $P$ & Odds ratio & $95 \% \mathrm{Cl}$ \\
\hline G & $\mathrm{T}$ & G & $30(26 \%)$ & $67(32 \%)$ & 0.31 & 0.745 & $0.434-1.273$ \\
\hline G & $\mathrm{T}$ & $A$ & $34(29 \%)$ & $56(27 \%)$ & 0.70 & 1.140 & $0.668-1.944$ \\
\hline $\mathrm{T}$ & $\mathrm{T}$ & $A$ & $16(14 \%)$ & $31(15 \%)$ & 0.94 & 0.924 & $0.458-1.851$ \\
\hline G & C & $A$ & $36(31 \%)$ & $56(27 \%)$ & 0.48 & 1.238 & $0.729-2.098$ \\
\hline
\end{tabular}

${ }^{a}$ HLA-DRB1 genotyping was available for $130 \mathrm{MS}$ patients and 155 controls.

$P$-value for the distribution of all four haplotypes in all MS vs all controls=0.91. $P$-value for the distribution of all four haplotypes in DRB1*1501-positive MS vs controls=0.45. P-value for the distribution of all four haplotypes in DRB1*1501-positive MS vs controls=0.65.

other SNP positions. In total, four haplotypes were observed (Figure 1), which were used to assign haplotypes to MS and control individuals.

Frequencies of these four haplotypes, based on genotyping of promoter region alleles, were compared in 176 MS cases and 176 control individuals (Table 3). All MS and control haplotypes were in Hardy-Weinberg equilibrium. Although $P$-values did not reach significance, there was a trend towards an increase of the GTG haplotype and a decrease in the TTA haplotype in the HLA-DRB1*1501 MS patients (Table 3 ).

\section{Estimation of IL-7R SNP allele frequencies from pooled DNA sequencing}

Pooled DNA sequencing was used to identify novel polymorphisms, but it also allows an approximation of allele frequency. In the sequence from an individual, the peak height at any position represents detection of $50 \%$ signal from each allele. In pooled samples, the peak height should also reflect the relative amounts of template for each SNP. This will not necessarily be quantitative, since there is spectral overlap between labelled ddNTPs, and the software correction may not be accurate through the range of signal detection. However, estimates of allele frequency from pooled DNA sequencing usually correlate well with the allele frequencies measured by other methods, for example, the frequency of $-504 \mathrm{C}$ was measured at $33 \%$ (controls) and 25\% (DR2+ MS) by PCR-RFLP, and 30\% for both from pooled DNA sequencing.

\section{Discussion}

In this study, 13 SNPs were detected in the IL-7R $\alpha$-chain gene in pooled and individual DNA samples, which were then investigated as candidate gene polymorphisms for MS susceptibility by genotyping Australian MS cases and control. Pooled DNA sequencing was found in this study to be a useful technique for rapidly screening the IL-7R gene for SNPs.

While IL-7R $\alpha$-chain gene mutations have been identified in several severe combined immunodeficiency patients, which have correlated with defective T-cell development, ${ }^{24,27}$ to date, no disease association studies with IL-7R polymorphisms have been reported. In all, 30 SNPs have been detected across the chromosome 5ptel-q13 region $^{28}$ by denaturing high-performance liquid chromatography, however none of these have been localised to the IL-7R $\alpha$ gene. In the present study, both coding and noncoding SNPs were identified. Noncoding SNPs, including those in the promoter and intronic sequences, have been identified in cytokine and other immunological genes and some of these have been found to be disease associated. $^{29}$ There was evidence that two of the IL-7R promoter SNPs potentially bind different transcription factors, including nuclear factor for activated $\mathrm{T}$ cells; however, the functional effects of these need to be further investigated.

The process of identifying haplotypes between SNPs known as 'haplotype-tagging', ${ }^{20}$ has been proposed as a faster method for locating potential disease-associated 
SNPs, as it can potentially reduce the amount of genotyping of individual SNPs by typing only those SNPS that define haplotypes. Also, it enables the disease-associated polymorphism to be determined more precisely. This has been demonstrated in a recent report of the IL- 8 gene and associations with bronchiolitis, ${ }^{30}$ where only one of two haplotypes containing the disease-associated -251A allele, was shown to be associated with the disease, indicating that the $-251 \mathrm{~A}$ polymorphism was not likely to be the disease-causing variant. Haplotype analysis of the IL-7R $\alpha$ chain gene in this study showed that there was linkage disequilibrium across the IL7-R region, with four haplotypes detected, which could be determined by genotyping just three out of the nine SNPs for which haplotype associations were known, substantially reducing the amount of genotyping required for determination of haplotype frequency. Furthermore, two of these haplotypes showed a trend towards positive or negative association with MS. Interestingly, this difference was observed when MS patients were stratified for DRB1*1501. Genome screening results have indicated that patterns of linkage and association between DRB1*1501-positive and -negative MS patients vary with different chromosomal regions, which points to a possible difference in gene usage in these individuals. Indeed, the chromosome 5cen region was found to be preferentially linked in DRB1*1501-negative MS patients, ${ }^{31}$ which was not supported by our results.

One remarkable feature of these novel IL-7R SNPs found in this study is that they are not rare. The method of detection used here is limited to those SNPs with an allele frequency of greater than $10-15 \%$. It is likely that there are many variants of IL7R less common than this, which will mark haplotypes rarer than those identified in this study, and which may be associated with MS. It is also likely that the variation in other genes, which have not been studied by sequencing of multiple individuals, is much greater than is often assumed. This could cause false rejection of disease associations, and it is certainly the case with IL7R, that an association between MS and any unknown, less common gene polymorphisms in IL7R cannot be excluded.

Overall, this study has identified 11 novel SNPs in the IL7R gene. We identified a haplotype (GTG) that was overrepresented in DRB1*1501-positive MS cases, and which warrants further investigation in MS and, given the key role of IL-7 and its receptor in T-cell development, in other autoimmune diseases. The effect of these SNPs on IL-7R function remains to be elucidated.

\section{Acknowledgements}

We thank Dr Susan Adams for the recruitment of MS cases, MS trio families, MS multiplex families and unrelated controls, Professor James McLeod and Claudia Charlton at the University of Sydney and Royal Prince Alfred Hospital for the recruitment of MS cases and unrelated controls, Dr Rex Simmons and Sandra Surkus at The Canberra Hospital for the recruitment of MS multiplex families, Mark Hibbins at the Department of Renal Medicine, Westmead Hospital and Peter Csurhes at Royal Brisbane Hospital for providing unrelated control samples, and Marc Buhler for preparing cell lines on MS patient and control samples. We acknowledge Natalie Hartley, Matthew Bugeja and Vanessa Perich for constructing the DNA pools, Natalie Hartley and Vanessa Perich for technical assistance with genotyping and DNA sequencing, Maria Ban for HLA-DRB1*1501 genotyping MS cases and MS trio probands used in the DNA pools, and the NSW Red Cross Tissue Typing Laboratory, Sydney for HLA-DR genotyping MS cases. This study was supported by The National Health and Medical Research Council of Australia and the Westmead Millennium Foundation.

\section{References}

1 Dyment DA, Sadnovich AD, Ebers GC: Genetics of multiple sclerosis. Hum Mol Genet 1997; 6: 1693-1698.

2 Sawcer S, Jones H, Feakes R et al: A genome screen in multiple sclerosis reveals susceptibility loci on chromosome 6p21 and 17q22. Nat Genet 1996; 13: 464-468.

3 Haines J, Pericak-Vance M, Seboun E, Hauser S, the Multiple Sclerosis Genetics Group: A complete genomic screen for multiple sclerosis underscores a role for the major histocompatibility complex. Nat Genet 1996; 13: 469-471.

4 Ebers GC, Kukay K, Bulman DE et al: A full genome search in multiple sclerosis. Nat Genet 1996; 13: 472-480.

5 Kuokkanen S, Gschwend M, Rioux JD et al: Genomewide scan of multiple sclerosis in Finnish multiplex families. Am J Hum Genet 1997; 61: 1379-1387.

6 Broadley S, Sawcer S, D'Alfonso S et al: A genome screen for multiple sclerosis in Italian families. Genes Immun 2001; 2: 205-210.

7 Coraddu F, Sawcer S, D'Alfonso S et al: A genome screen for multiple sclerosis in Sardinian multiplex families. Eur J Hum Genet 2001; 9: 621-626.

8 Stewart GJ: Multiple sclerosis, multiple genes. Med J Aust 1997; 166: $573-574$

9 Oturai A, Larsen F, Ryder LP et al: Linkage and association analysis of susceptibility regions on chromosomes 5 and 6 in 106 Scandinavian sibling pair families with multiple sclerosis. Ann Neurol 1999; 46: 612-616.

10 Sundvall M, Jirholt J, Yang HT et al: Identification of murine loci associated with susceptibility to chronic experimental autoimmune encephalomyelitis. Nat Genet 1995; 10: 313-317.

11 Kuokkanen S, Sundvall M, Terwilliger JD et al: A putative vulnerability locus to multiple sclerosis in a region syntenic to the murine locus Eae2. Nat Genet 1996; 13: 477-480.

12 Chataway J, Sawcer S, Sherman D et al: No evidence for association of multiple sclerosis with the complement factors C6 and C7. J Neuroimmunol 1999; 99: 150-156.

13 Pleiman CM, Gimpel SD, Park LS, Harada H, Taniguchi T, Ziegler SF: Organization of the murine and human interleukin-7 receptor genes: two mRNAs generated by differential splicing and presence of a type I-interferon-inducible promoter. Mol Cell Biol 1991; 11: 3052-3059.

14 Plum J, De Smedt M, Leclercq G, Verhasselt B, Vandekerckhove B: Interleukin-7 is a critical growth factor in early human T-cell development. Blood 1996; 88: 4239-4245.

15 Ziegler SF, Morella KK, Anderson D et al: Reconstitution of a functional interleukin (IL)-7 receptor demonstrates that the IL-2 receptor $\gamma$ chain is required for IL-7 signal transduction. Eur J Immunol 1995; 25: 399-404.

16 Chou YK, Bourdette DN, Barnes D et al: IL-7 enhances Ag-specific human T cell response by increasing expression of IL-2R $\alpha$ and $\gamma$ chains. J Neuroimmunol 1999; 96: 101-111. 
17 Traggiai E, Biagioli T, Rosati E et al: IL-7 enhanced T-cell response to myelin proteins in multiple sclerosis. J Neuroimmunol 2001; 121: 111-119.

18 Bebo Jr BF, Shuster JC, Adlard K, Vandenbark AA, Offner H: Interleukin 7 is a potent co-stimulator of myelin specific $\mathrm{T}$ cells that enhances the adoptive transfer of experimental autoimmune encephalomyelitis. Cytokine 2000; 12: 324-331.

19 Ramanathan M, Weinstock-Guttman B, Nguyen LT et al: In vivo gene expression revealed by cDNA arrays: the pattern in relapsing-remitting multiple sclerosis patients compared with normal subjects. J Neuroimmunol 2001; 116: 213-219.

20 Johnson GC, Esposito L, Barratt BJ et al: Haplotype tagging for the identification of common disease genes. Nat Genet 2001; 29: 233-237.

21 Tabor HK, Risch NJ, Myers RM: OPINION: candidate-gene approaches for studying complex genetic traits: practical considerations. Nat Rev Genet 2002; 3: 391-397.

22 Poser CM, Sheinberg L, McDonald WI et al: New diagnostic criteria for multiple sclerosis: guidelines for research protocols. Ann Neurol 1983; 13: 227-231.

23 Barcellos LF, Klitz W, Field LL et al: Association mapping of disease loci, by use of a pooled DNA genomic screen. Am J Hum Genet 1997; 61: 734-747.

24 Puel A, Ziegler SF, Buckley RH, Leonard WJ: Defective IL7R expression in $\mathrm{T}(-) \mathrm{B}(+) \mathrm{NK}(+)$ severe combined immunodeficiency. Nat Genet 1998; 20: 394-397.
25 Spielman RS, McGinnis RE, Ewens WJ: Transmission test for linkage disequilibrium: the insulin gene region and insulindependent diabetes mellitis (IDDM). Am J Hum Genet 1993; 52: 506-516.

26 Wingender E, Chen X, Hehl R et al: TRANSFAC: an integrated system for gene expression regulation. Nucleic Acids Res 2000; 28: 316-319.

27 Roifman CM, Zhang J, Chitayat D, Scharfe N: A partial deficiency of interleukin-7R $\alpha$ is sufficient to abrogate T-cell development and cause severe combined immunodeficiency. Blood 2000; 15: 2803-2807.

28 Giordano M, Oefner PJ, Underhill PA, Cavali Sforza LL, Tosi R, Momigliano Richiardi P: Identification by denaturing highperformance liquid chromatography of numerous polymorphisms in a candidate region for multiple sclerosis susceptibility. Genomics 1999; 56: 247-253.

29 Mitchison NA: Polymorphism in regulatory gene sequences. Genome Biol 2001; 2: 1-6.

30 Hull J, Ackerman $\mathrm{H}$, Isles $\mathrm{K}$ et al: Unusual haplotypic structure of IL8, a susceptibility locus for a common respiratory virus. Am J Hum Genet 2001; 69: 413-419.

31 Coraddu F, Sawcer S, Feakes R et al: HLA typing in the United Kingdom multiple sclerosis genome screen. Neurogenetics 1998; 2: 24-33. 